\title{
Das Zentrum für Hautkrankheiten am Klinikum Stuttgart
}

\author{
The Center for Dermatological Diseases at the Klinikum Stuttgart
}

Autor

Institut

\section{P. von den Driesch}

Klinik für Dermatologie und Allergologie (Chefarzt: Prof. Dr. med. P. von den Driesch), Zentrum für Hautkrankheiten, Klinikum Stuttgart

\section{Bibliografie}

Dol $10.1055 / \mathrm{s}-2006-944938$ Akt Dermatol 2006; 32; 507-510 @ Georg Thieme Verlag KG Stuttgart · New York ISSN 0340-2541

Korrespondenzadresse Prof. Dr. med. Peter von den Driesch

Klinik für Dermatologie und Allergologie, Zentrum für Hautkrankheiten · Klinikum Stuttgart Prießnitzweg 24 70374 Stuttgart pdriesch@kbc-intern.de

\section{Zusammenfassung \\ $\nabla$}

Die im Jahre 1837 von Albert Friedrich Veiel in Cannstatt gegründete „Heilanstalt für Flechtenkranke“ gilt als erste Hautklinik Deutschlands. Nach wechselvoller Geschichte besteht an diesem Ort jetzt das „Zentrum für Hautkrankheiten“ am Klinikum Stuttgart, einem Haus der Maximalversorgung mit 2400 Betten in 52 Fachabteilungen. Das Zentrum für Hautkrankheiten besteht aus der „Klinik für Dermatologie und Allergolo-

\section{Dermatologie in Stuttgart-Bad Cann- statt: historische Aspekte \\ $\nabla$}

Der Beginn der klinischen Dermatologie in Stuttgart-Bad Cannstatt fällt in das Jahr 1837, als der aus Ludwigsburg stammende Arzt Albert Friedrich Veiel (1806-1874) die private „Heilanstalt für Flechtenkranke“ und damit die erste Hautklinik Deutschlands gründete [1]. 1879-1881 errichtete die Amtskörperschaft Cannstatt nach seinen Vorgaben das Krankenhaus Bad Cannstatt als Bezirkskrankenhaus. Erfahrungen des Feldzuges 1870/1871 waren hierbei die Ursache, das Krankenhaus in der Nähe eines Schienenanschlusses zu bauen, der auch heute noch die gute Erreichbarkeit der Klinik für die Patienten ermöglicht.

Albrecht Friedrich Veiel und seine Söhne Ernst Veiel (1837-1883) und Theodor Veiel (1848-1923) sowie der Enkel Fritz Veiel leiteten das Krankenhaus anschließend bis 1935. Mit der am 18.1.1935 eröffneten, neu erbauten Hautklinik knüpfte das Krankenhaus Bad Cannstatt dann an seine dermatologische Tradition an. Erste klinische Leiter waren Erich Schmidt (1888-1967) und Clemens Hövelborn

\footnotetext{
${ }^{*}$ Herrn Prof. Dr. O. P. Hornstein zum 80. Geburtstag gewidmet.
}

gie“, geleitet von Prof. Dr. med. Peter von den Driesch, und der „Klinik für Dermatologie und Phlebologie“, geleitet von Priv.-Doz. Dr. med. Jörg Keller. Beide Kliniken verfügen über 99 vollstationäre und 21 teilstationäre Betten und betreuen etwa 4500 vollstationäre und 700 teilstationäre Patienten pro Jahr. In dem 1998 vollständig renovierten und hochmodernen Zentrum steht das gesamte dermatologische Leistungsspektrum zur Verfügung.

(1905-1994). Bei der Neugestaltung waren wesentliche Impulse von Prof. Dr. W. Sevin eingebracht worden, welcher seine Tätigkeit für die Stadt Stuttgart aus politischen Gründen (seine Ehefrau war Jüdin) 1936 unterbrechen musste. Auffällig ist die von Sevin gewählte Z-Form: ein Männerbau und ein Frauenbau waren verbunden und zugleich getrennt durch ein Behandlungsgebäude ( $\bullet$ Abb. 1).

Das im Zweiten Weltkrieg weitgehend verschont gebliebene Krankenhaus Bad Cannstatt wurde am 8.8.1945 von der amerikanischen Militärregierung beschlagnahmt und Wilhelm Sevin (1900-1976) übernahm die ärztliche Leitung der allerdings in verschiedene Quartiere ausgelagerten Hautklinik. Erst im Jahre 1965 konnte nach hartnäckigen Verhandlungen durch Prof. Sevin die Hautklinik in ihr ursprüngliches Gebäude zurückkehren.

1967 wurde Wilhelm Knoth zum Ärztlichen Direktor der 278 Betten umfassenden Hautklinik Stuttgart-Bad Cannstatt berufen. In den folgenden Jahren konnte der engagierte und bekannte Dermatologe ungerechtfertigte Verkleinerungen der Klinik, deren Einzugsgebiet über 4 Millionen Einwohner umfasst, verhindern. 1971 kam die Gründung einer eigenen Allergologie-Abteilung unter Prof. Dr. Dieter Kleinhans hinzu. 


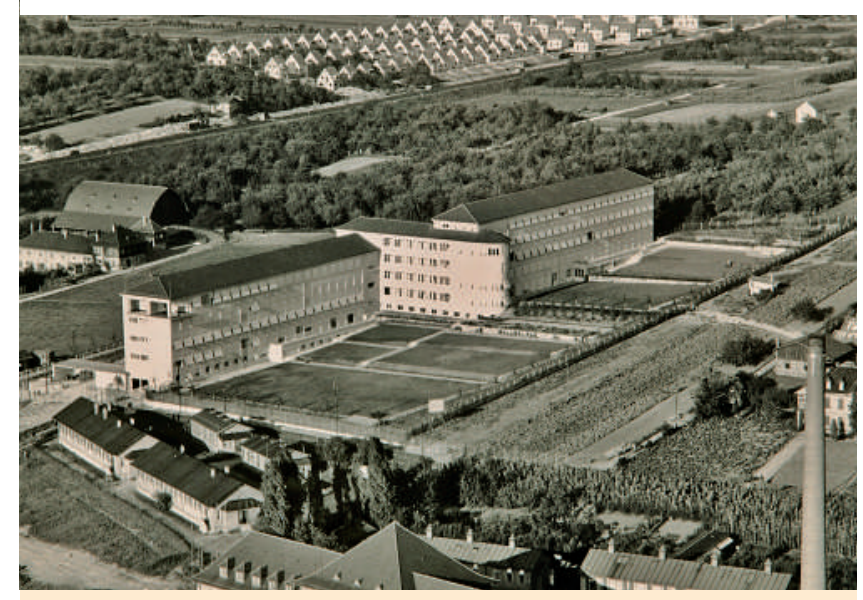

Abb. 1 Fotografie der 1935 erbauten Hautklinik Bad Cannstatt mit ihrem charakteristischen Z-Aufbau. Die Klinik besaß 261 Betten, ein Männer- und ein Frauentrakt waren getrennt und gleichzeitig verbunden durch die in der Mitte positionierten Behandlungsräume.

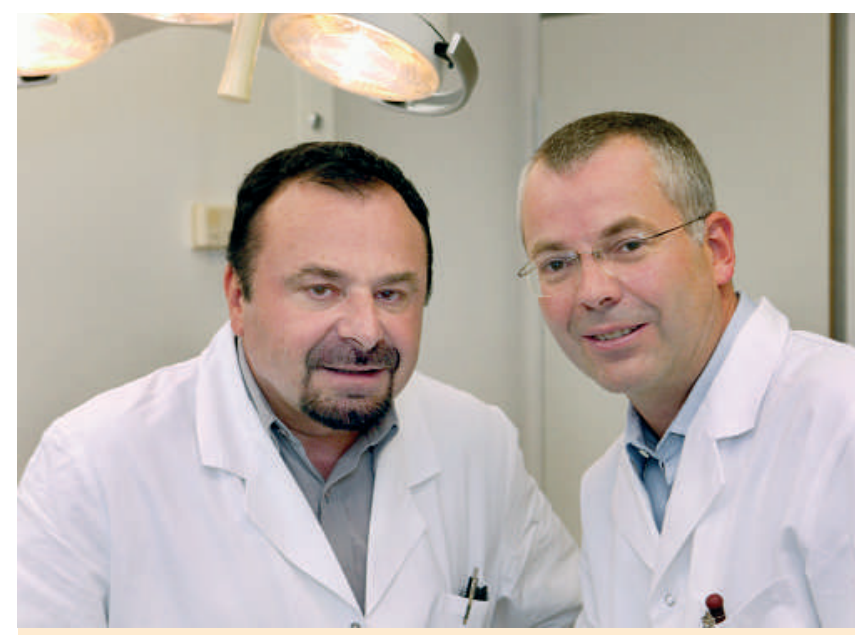

Abb. 3 Die beiden Ärztlichen Direktoren des Zentrums für Hautkrankheiten am Klinikum Stuttgart: Prof. Dr. P. von den Driesch (rechts) und Priv.-Doz. Dr. J. Keller (links).

Nach dem plötzlichen Tod von Wilhelm Knoth am 11. April 1982 wurde die Ärztliche Direktion der Hautklinik an den Schüler von Otto Braun-Falco und Otto P. Hornstein Frank O. Weidner übertragen, zu diesem Zeitpunkt mit noch 138 Betten. Im November 1992 wurde unter der ärztlichen Leitung von Priv.-Doz. Dr. med. Jörg Keller ( $\bullet$ Abb. 3), ebenfalls Schüler von Otto P. Hornstein, die Abteilung für „Dermatologie und Phlebologie“ gegründet, aus der 1998 die Klinik für Dermatologie und Phlebologie entstand.

Nachdem im September 1992 Teile des Krankenhausgebäudes von der US-Armee freigegeben wurden, konnte bis zum Herbst 1998 das nun vollständig renovierte Zentrum für Hautkrankheiten mit 99 vollstationären und 21 teilstationären Betten realisiert werden ( $\bullet$ Abb. 2). Damals wurde das Zentrum für Hautkrankheiten mit den beiden selbständigen Kliniken „Klinik für Dermatologie und Allergologie (Prof. Dr. F. O. Weidner) und Klinik für Dermatologie und Phlebologie (Priv.-Doz. Dr. J. Keller)“ gegründet.

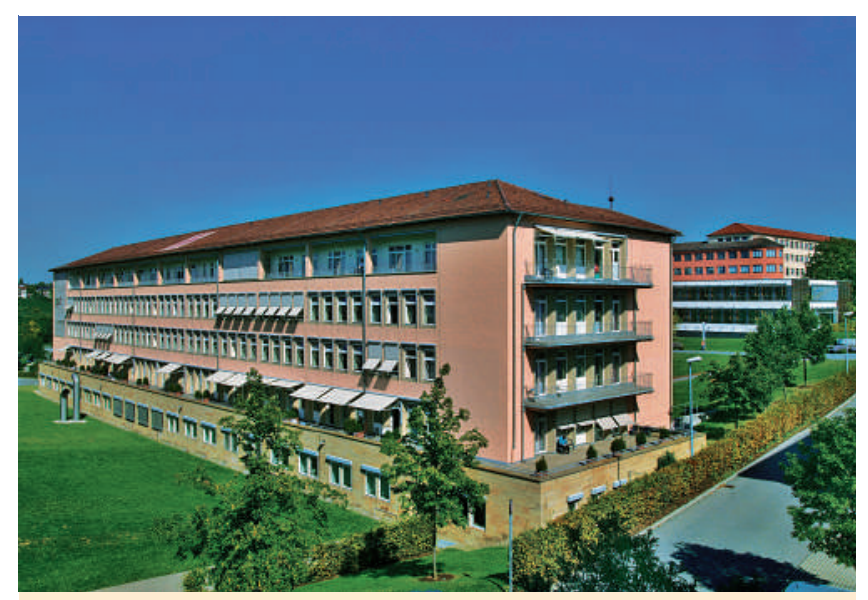

Abb. 2 Das Zentrum für Hautkrankheiten im Jahre 2006. Das 1935 erbaute Gebäude wurde vollständig geräumt (zur Orientierung: der ehemalige Behandlungstrakt entspricht dem im Hintergrund sichtbaren rot gefärbten Gebäudeteil). Das neue bis 1998 erbaute Zentrum bietet 99 vollstationäre und 21 teilstationäre Betten mit allen notwendigen Behandlungsmöglichkeiten.

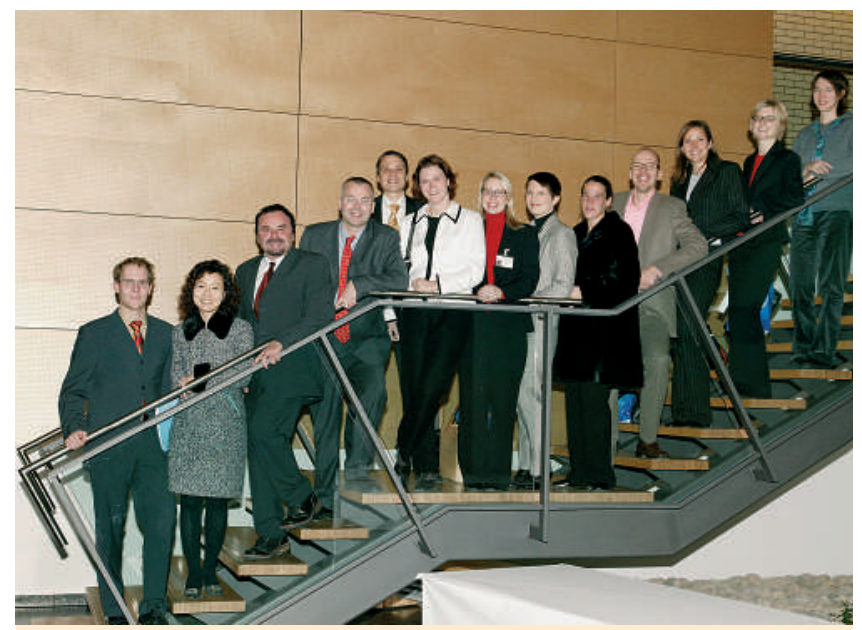

Abb. 4 Die ärztlichen Direktoren mit Assistenten anlässlich der Tagung der Vereinigung Württembergischer Dermatologen in Cannstatt.

Zum 1. 7. 2003 übernahm Herr Prof. Dr. Peter von den Driesch ( $\bullet$ Abb. 3), Schüler von Otto P. Hornstein und vorher C3-Professor am Universitätsklinikum Eppendorf in Hamburg unter der Leitung von Frau Prof. Dr. I. Moll, die Ärztliche Leitung der Klinik für Dermatologie und Allergologie. Die von Prof. Dr. D. Kleinhans aufgebaute und nach ihm von Dr. Hartmut Finkbeiner geleitete Abteilung für Allergologie wurde wieder reintegriert.

\section{Schwerpunkte der klinischen Arbeit \\ $\nabla$}

Entsprechend den Anforderungen an ein modernes Zentrum für Hautkrankheiten decken die beiden Kliniken nun das gesamte Gebiet der Dermatologie, Venerologie, Allergologie, Phlebologie, Proktologie und ästhetische Chirurgie ab. Das Zentrum für Hautkrankheiten stellt mit 99 vollstationären Betten und 21 teilstationären Betten, 4500 vollstationären Patienten, 700 teilstationären Patienten und 25000 ambulanten oder konsiliarisch gese- 


\begin{tabular}{|c|c|c|c|c|c|c|c|}
\hline & \multirow{2}{*}{$\begin{array}{l}\text { vollstat. } \\
\text { Betten }\end{array}$} & \multirow{2}{*}{$\begin{array}{l}\text { teilstat. } \\
\text { Betten }\end{array}$} & \multicolumn{3}{|c|}{ vollstat. Patienten } & \multirow[t]{2}{*}{ teilstat. Pat. } & \multirow{2}{*}{$\begin{array}{l}\text { Tab. } 1 \text { Eckdaten des } \\
\text { Zentrums für Hautkrankheiten } \\
\text { am Klinikum Stuttgart }\end{array}$} \\
\hline & & & 2003 & 2004 & 2005 & & \\
\hline Zentrum für Hautkrankheiten & 99 & 21 & 3774 & 4385 & 4509 & 681 & \\
\hline $\begin{array}{l}\text { Klinik für Dermatologie und } \\
\text { Allergologie }\end{array}$ & 50 & 10 & 1846 & 2122 & 2283 & 346 & \\
\hline $\begin{array}{l}\text { Klinik für Dermatologie und } \\
\text { Phlebologie }\end{array}$ & 49 & 11 & 1958 & 2263 & 2226 & 335 & \\
\hline
\end{tabular}

henen Patienten eine der größten Hautkliniken Deutschlands dar. Derzeit konnten trotz des allgegenwärtigen ökonomischen Drucks weitere Schließungen von Betten erfolgreich verhindert werden.

Das gesamte dermatologisch-diagnostische Spektrum einschließlich Histologie, Immunhistologie, allergologische, andrologische und phlebologische Diagnostik steht zur Verfügung. In zwei, nur für die Hautkliniken zur Verfügung stehenden Operationssälen werden etwa 3000 klassische dermatochirurgische Eingriffe, 591 ästhetisch-chirurgische Operationen und 1210 Eingriffe am Venenapparat durchgeführt.

Klinische Schwerpunkte sind die dermatologische Onkologie und operative Dermatologie, das Ulcus cruris in seinen verschiedenen Erscheinungsformen, klassische Dermatosen wie schwere Verläufe von Psoriasis, Ekzem-Erkrankungen, Lichen ruber und Pityriasis rubra pilaris sowie die Therapie autoimmun bedingter blasenbildender Erkrankungen und Kollagenosen.

Individuelle Schwerpunkte sind in der Klinik für Dermatologie und Allergologie die Diagnose und Behandlung von Insektengiftallergien, Arzneimittelallergien, Berufsdermatosen, Nahrungsmittelallergien und inhalativen Allergien sowie in der Klinik für Dermatologie und Phlebologie die Diagnostik und operative Therapie von Venenleiden sowie alle Aspekte der ästhetischen Dermatologie.

Die klassische lokale Dermatotherapie wird durch Lichttherapie (UVA, PUVA, Bade-PUVA, UVA-1, UVB, UVB-311 nm) sowie moderne physikalische Therapieverfahren einschließlich photodynamischer Therapie unterstützt. Bezüglich der systemischen Dermatotherapie werden auch modernste immunologische Ansätze wie intravenöse Immunglobulin-Gabe oder Rituximab verfolgt. Diese aufwendigen Therapieverfahren werden insbesondere in der Therapie dermatologischer Autoimmunerkrankungen und der Vaskulitis bzw. vaskulitischer Ulzera mit Erfolg eingesetzt. In der Dermatoonkologie stehen alle gängigen Verfahren der Mono- und Polychemotherapie sowie neuere Antikörper-basierte Modalitäten zur Verfügung. In der Hyposensibilisierung von Bienen- und Wespengift-Allergikern steht eine schnelle 2-tägige stationäre Einleitung der Therapie zur Verfügung, die bei mehr als 150 Patienten im Jahr mit dieser Indikation erfolgreich eingesetzt wird. Im Bereich Venentherapie kommen alle klassischen operativen Verfahren, „Venous closure“, Schaumsklerosierung und Lasertechniken zum Einsatz, in der ästhetischen Dermatologie korrigierende Eingriffe im Gesicht (z. B. Lifting, Lidstraffung) sowie Liposuktion verschiedener Körperareale und die axilläre Schweißdrüsen-Saugkürretage.

In den letzten Jahren hat das Zentrum für Hautkrankheiten seine Anstrengungen im Bereich der Erkennung und Behandlung vaskulitischer Ulzera intensiviert. Hier wird nach einem standardisierten Schema die Diagnose gesichert und eine multimodale immunologische Therapie einschließlich von intravenösen Immunglobulinen eingesetzt. Unterstützt wird dies durch alle
Tab.2 Sprechstunden des Zentrum für Hautkrankheiten am Klinikum Stuttgart

Klinik für Dermatologie und Allergologie

Allgemeine Sprechstunde

Allergologische und berufsdermatologische Sprechstunde

Onkologische Sprechstunde

Ulkussprechstunde

Klinik für Dermatologie und Phlebologie

Allgemeine Sprechstunde

Phlebologische Sprechstunde

Proktologische Sprechstunde

Ästhetisch-dermatologische Sprechstunde

gängigen Verfahren der Ulkusreinigung, beispielsweise mittels Wasserstrahlskalpell $\left(\right.$ Versajet $\left.^{\circledR}\right)$, und einer phasengerechten Wundtherapie in einer spezialisierten Sprechstunde unter Hinzuziehung eines qualifizierten Wundmanagers.

\section{Klinische Forschung und Publikationen \\ $\nabla$}

Entsprechend den Aufgaben und der Arbeitsintensität des Zentrums steht die klinische Forschung im Vordergrund [2-8]. Besonderes, auch wissenschaftliches Interesse hat das Zentrum auf dem gesamten Gebiet der Dermatoonkologie, insbesondere der Therapie kutaner Lymphome, der Anwendung neuerer operativer Verfahren (Dermatochirurgie, ästhetische Chirurgie, Venenchirurgie), Diagnose und Therapie der Vaskulitis einschließlich der sog. vaskulitischen Ulzera sowie der Therapie der blasenbildenden Erkrankungen und Kollagenosen.

\section{Fortbildung}

$\nabla$

Neben der regelmäßigen internen Fortbildungsveranstaltungen führt das Zentrum regelmäßig Themenabende mit klinisch relevanten Inhalten durch. Eine langjährige Tradition sind die Sitzungen der Vereinigung Württembergischer Dermatologen, welche im Sommer durch die Universitäts-Hautklinik Tübingen, stets am ersten Wochenende im Dezember durch das Zentrum für Hautkrankheiten in Stuttgart durchgeführt werden (- Abb. 4). 2007 wird, erstmals nach der erfolgreichen Durchführung der 102. Tagung der Südwestdeutschen DermatologenVereinigung 1972 durch Prof. Dr. W. Knoth, unter der Leitung von Prof. Dr. P. von den Driesch und Priv.-Doz. Dr. J. Keller die 134. Tagung der Vereinigung Südwestdeutscher Dermatologen zusammen mit der 95. Sitzung der Vereinigung Württembergischer Dermatologen vom 30. November bis 1. Dezember im Kultur- und Kongresszentrum Liederhalle in Stuttgart durchgeführt. 
Abstract

\section{The Center for Dermatological Diseases at the Klinikum Stuttgart}

In 1837 Albert Friedrich Veiel founded the „Heilanstalt für Flechtenkranke“ in Cannstatt, Germany, which is said to be the first dermatological hospital in Germany. After an impressive history, the „Center for Dermatology“ today belongs to the „Klinikum Stuttgart“, a hospital complex offering maximum medical treatment with its 52 different departments and 2400 beds. The Center for Dermatology consists of the Clinic for Dermatology and Allergology, headed by Prof. Dr. Peter von den Driesch, and the Clinic for Dermatology and Phlebology, headed by Priv.-Doz. Dr. J. Keller. Both clinics have 99 beds for in-patients and 21 beds for day-patients. In this centre, which was completely renovated in 1998, the full range of dermatological treatment is available.

\section{Literatur}

1 Finkbeiner H, Weidner FO. Die Hautklinik des Krankenhauses Bad Cannstatt - Ursprünge und Entwicklung der klinischen Dermatologie in Stuttgart. Hautarzt 1997; 48: 762 - 765

2 Coors EA, Wessbecher $R$, von den Driesch P. Beastly nodules instead of beauty: pseudoepitheliomatous hyperplasia developing after application of permanent make-up. Br J Dermatol 2004; 150: 1027

3 Coors EA, von den Driesch P. Topical photodynamic therapy for patients with therapy-resistant lesions of cutaneous T-cell lymphoma. J Am Acad Dermatol 2004; 50: 363-367

4 Coors EA, von den Driesch P. Treatment of mycosis fungoides with bexarotene and psoralen plus ultraviolet A. Br J Dermatol 2005; 15: $1379-1381$

5 Coors EA, Schuler G, von den Driesch P. Topical imiquimod as treatment for different kinds of cutaneous lymphoma. Eur J Dermatol 2006; 16: $391-393$

6 Kaufmann R, Spieth K, Leiter U, Mauch C, von den Driesch P et al. Temozolomide in combination with interferon-alfa versus temozolomide alone in patients with advanced metastatic melanoma: a randomized, phase III, multicenter study from the Dermatologic Cooperative Oncology Group. J Clin Oncol 2005; 23: 9001 - 9007

7 Modschiedler K, von den Driesch P, Paus R. Hyperpigmentierung und Hypertrichose der Augenlider nach Bimatoprost Augentropfen. JDDG 2005; 3: $276-277$

8 Stetter C, Plaza T, von den Driesch P. Skin grafting of a chronic leg ulcer with combined Versajet ${ }^{\circledR}$-V.A.C. therapy. JDDG 2006; 4: 739-742

\section{Buchbesprechung}

Presse-Taschenbuch Gesundheit 2006/07

Bayer HealthCare (Hrsg.)

Seefeld: Kroll-Verlag, 2006. 704 S. Kart. 32,00€

ISBN 3-936797-26-9

Das Presse-Taschenbuch Gesundheit bietet mit seiner Neuauflage 2006/2007 im 34. Jahr seines Bestehens eine kompakte Informationssammlung von Namen und Adressen aus den Bereichen Medizin und Gesundheit. Es wendet sich primär an Journalisten bzw. Personen aus den Bereichen Medien und Kommunikation. Das handliche, flexibel eingebundene Büchlein, 704 Seiten Dünndruckpapier in DIN-A 6-Format, ist mit gut 2 Zentimeter Dicke tatsächlich westentaschenfähig. Die Auflistung startet mit dem who is who der Medizin-Nobelpreisträger, gefolgt von Medizin und Wissenschaftsjournalisten mit Adressen, Telefon- und Faxnummern sowie zahlreichen Internet- und E-Mail-Adressen.

Publikationen und elektronische Medien sind mit den Medizinredaktionen der Tages- und Wochenpresse bis zu medizinischen Fachbuchverlagen, Funk und Fernsehen vertreten. Der Absatz „Wissenschaft und Forschung“ nennt öffentliche und private bzw. Stiftungseinrichtungen. Im Absatz „Behörden, Bund und Länder“ finden sich vom Bundespräsidenten bis zu den Landesbehörden Ansprechpartner der Bundes- und Landespolitik. Schließlich umfasst der größte Absatz (knapp 200 Seiten) mit Verbänden und Organisationen Organe der Ärtzeschaft, der Zahnärzte und verwandten Berufe, darüber hinaus Verbände und wissenschaftliche Organisationen. Ein alphabetisches Namensverzeichnis am Ende erleichtert den direkten namentlichen Zugriff. Bayer HealthCare als Sponsor hält sich mit 15 Seiten (den einzigen farbigen) am Ende des Büchleins relativ im Hintergrund.
Kann der nicht hauptsächlich journalistisch tätige Arzt in Klinik oder Praxis vom Presse-Taschenbuch profitieren? Ja „nicht immer, aber immer öfter“: Teile der Adresssammlung sind im engeren Sinne auch für die tägliche ärztliche Situation sinnvoll einsetzbar, zumal in Zeiten massiver politischer Umbrüche im Gesundheitswesen und individueller oder organisierter Willensbekundungen aus der Ärzteschaft. Hierfür finden sich zielgerichtet die korrekten Ansprechpartner. Der Einfluss der Medien auf die Arzt-Patient-Beziehung legt auch den praktizierenden Medizinern nahe, die laien-orientierten Gesundheitssendungen der Fernsehsender zu verfolgen, um den Informationsstand ihrer Patienten abschätzen und auf Fragen Antworten parat haben zu können. Als weiterer Teil des PresseTaschenbuchs ist die umfassende Adresssammlung von Selbsthilfegruppen zu begrüßen. Hier kann den Patienten mit konkreten Hinweisen auf gleichartig Betroffene weitergeholfen werden.

Zusammenfassend sind Hautärzte wie auch Mediziner anderer Fachrichtungen zwar nicht primäre Adressaten des Büchleins, Nutzungsmöglichkeiten ergeben sich aber aus Aktualität und Vielfalt dieses Verzeichnisses. Interessant noch die Information, dass eine digitale Version des Presse-Taschenbuchs nicht allgemein zugänglich ist, der Verlag aber seine Hilfe bei der Zusammenstellung elektronischer Adressdateien aus gewünschten Kapiteln nach individuellen Bedürfnissen anbietet.

D. und B. Krahl, Heidelberg 